\title{
Lessons learned from the introduction of personalized genotyping into a medical school curriculum
}

\author{
David R. Walt, PhD ${ }^{1}$, Amy Kuhlik, $M D^{2}$, Scott K. Epstein, $M D^{2}$, Laurie A. Demmer, $M D^{3}$, \\ Meredith Knight, $M A^{1}$, David Chelmow, $M D^{3}$, Michael Rosenblatt, $M D^{2}$, and Diana W. Bianchi, $M D^{3}$
}

\begin{abstract}
Purpose: There is an expanding gap between the availability of directto-consumer whole genome testing and physician knowledge regarding interpretation of test results. Advances in the genomic literacy of health care providers will be necessary for genomics to exert its potential to affect clinical practice. However, implementation of a major shift in medical education to include genomics is not easily done. The purpose of this educational report is to describe efforts to incorporate knowledge of personalized medicine into a medical school curriculum. Methods: In this report, we describe the experiences, both good and bad, of a multidisciplinary faculty group that examined ways to improve genomic education at Tufts University School of Medicine during a 16-month period. Results: The results of the faculty's deliberation process resulted in the use of anonymous, rather than student genomes, to teach material on genomic medicine. Conclusion: Increased medical school education regarding genomic analysis and personalized medicine is a necessity, both to be able to translate the advances made by the Human Genome Project into improvements in human health and to begin to think of diseases as disruptions in specific pathways. Our experiences illustrate that adding this material to a medical school curriculum is a complex process that deserves careful thought and broad discussion within the academic community. Genet Med 2011:13(1):63-66.
\end{abstract}

Key Words: genomics, medical education, personalized medicine, ethics

In a recent Student Forum article published in PLoS Medicine, Salari, ${ }^{1}$ a Stanford medical student, eloquently described the expanding gap between the availability of direct-to-consumer whole genome testing and physician knowledge regarding interpretation of test results. She advocated for medical trainees to be given a strong educational foundation in genetics and genomics. Similarly, Guttmacher et al. ${ }^{2}$ described the differences between biomedical researchers and clinicians in their respective interpretations of the significance of the "genome era." They concluded that advances in the genomic literacy of health care providers will be necessary for genomics to exert its potential to affect clinical practice. We, as faculty members of Tufts University, agree with these recommendations. However, implementation of a major shift in medical education to incorporate genomics is not easily done. In the following article, we describe our efforts during a 16-month period to incorporate

From the ${ }^{1}$ Department of Chemistry, Tufts University, Medford; ${ }^{2}$ Department of Medicine and the Dean's Office, Tufts University School of Medicine, Boston; and ${ }^{3}$ Division of Genetics, Department of Pediatrics, Floating Hospital for Children at Tufts Medical Center, Boston, Massachusetts.

Diana W. Bianchi, MD, Tufts Medical Center, 800 Washington Street, Box 394, Boston, MA 02111. E-mail: Dbianchi@tuftsmedicalcenter.org.

Disclosure: The authors declare no conflict of interest

Submitted for publication June 8, 2010.

Accepted for publication August 19, 2010.

Published online ahead of print November 4, 2010.

DOI: 10.1097/GIM.0b013e3181f872ac knowledge of genomic medicine into the Tufts medical school curriculum. We believe that a description of our experiences, both good and bad, may be educational and informative for other programs contemplating integration of human genomic data into a clinical curriculum.

\section{THE IDEA}

In October 2008, while attending a Howard Hughes Medical Institute meeting, David R. Walt (D.R.W.) heard Sharon Long, $\mathrm{PhD}$, Vice-Chair of the American Association of Medical Colleges-Howard Hughes Medical Institute Scientific Foundations for Future Physicians Committee, discussing new requirements for both premedical and undergraduate medical students. One of the competencies that she mentioned for medical students was an understanding of personal genetic information. She stated that with the advent of inexpensive genetic testing, future physicians should be prepared to interpret the results of these tests. The committee also recommended that graduating physicians should have an understanding of gene-environment interactions and the statistical analytic techniques necessary to comprehend the probabilities associated with these studies.

As background information, D.R.W. was directly involved in the development of microarray technology. He also serves as a Director of Illumina, a company that develops microarrays and equipment to perform second generation deoxyribonucleic acid sequencing. It occurred to him that introducing a personal genetics component into the medical school curriculum would offer an exceptional opportunity for students to learn first hand about the process and would enable them to be trained to evaluate the analytic and clinical validity, as well as the clinical utility of the data. He reasoned that the Tufts medical students' own personal genotypes could serve as a backdrop for teaching issues related to clinical implementation, including the potential benefits and harms of these tests.

Knowing that genotyping 200 students per year would be costly and that coordinating such a project might be logistically complex, D.R.W. engaged 23andme, one of the companies involved in direct-to-consumer genotyping; 23andme uses Illumina microarrays as their platform. Linda Avey, one of the two founders of 23 andme, took an immediate interest in the idea. Educating future physicians is an important component of 23andme's business strategy, and they reasoned that introducing the idea at the earliest stage of medical education would help to educate future physicians regarding the power of personal genetics. After several phone calls and e-mail exchanges, 23 andme agreed to discount pricing for the medical students, essentially reducing the cost by their profit margin.

\section{THE INITIAL CONVERSATION}

To implement testing, D.R.W. met with the (then) Dean of Tufts Medical School (M.R.), and the Deans for Education (S.E.) and Student Affairs (A.K.) in November 2008. The 
"Dean's Team" was enthusiastic, especially because Tufts School of Medicine would be initiating an entirely new curriculum that emphasized translational medicine in fall of 2009. However, the Deans immediately raised concerns about how the information might adversely affect students. In particular, they were concerned about the impact on student mental health if an abnormality was discovered. They also raised the issue of the need for institutional review board (IRB) approval, informed consent, and the need to protect privacy. The decisions from the Deans' initial meeting were the following:

1. A pilot program would be implemented beginning in January 2009. The program would be explained to the current first-year students, and a subset (20-30 students) would be invited to volunteer for the pilot study. Issues with this smaller group of students would be explored to avoid any surprises and potential catastrophes if the entire class was involved from the start.

2. The students participating in the pilot study would meet regularly during the semester to learn about technology, genome-wide association studies, ethics, statistics, and data quality.

3. The full program would start with the fall 2009 entering class. That way, the 23 andme results could be integrated into the curriculum on the regular academic schedule.

\section{THE BROADER CONVERSATION}

A.K. then drafted a project description for the initial project committee. Before it was distributed, it was shown to the Chair of the Curriculum Committee (L.A.D.), who is herself a medical geneticist. She then distributed the draft to her colleagues in medical genetics (among them, D.W.B.), who expressed concern that this was being implemented without a full consideration of all the ethical implications. In addition to the concerns previously raised by the "Dean's Team," the clinical geneticists were hesitant to expose students to this technology that, while already commercially available, had not undergone rigorous evaluation for clinical validity and utility. A series of e-mail messages followed, which led to the formation of an informal committee to discuss personalized genomic testing of medical students. This committee, composed of the authors of this article, first met in January 2009 and raised the following issues:

1. What are the psychological risks of disclosing personal genetic information to students? In particular, would knowing that they had an abnormality affect learning in general, by causing students to focus on the conditions for which they might be at risk?

2. What is the privacy and confidentiality of the information disclosed? What are the unintended consequences for family members of the students tested?

3. Who would provide genetic counseling and medical follow-up for students who received abnormal results?

4. Was IRB approval needed for such a project?

5. What are the potential risks to students in presenting personal genome data that are lacking evidence for clinical validity and clinical utility?

One suggestion that came out of the initial meeting was to consider using anonymous genomes as an educational vehicle. This strategy would remove concerns regarding privacy, psychological impact, and risk of harm from potentially invalid data. However, some members of the committee maintained that receiving the results from one's personal genome would make the experience more meaningful.

\section{THE CONTROVERSY}

A draft IRB protocol for a pilot study using a subset of students was prepared. It included the rationale for the pilot study and how the information would be used as part of the educational curriculum. It would be clear that participation in this study would be completely voluntary, and students opting out would not suffer any adverse consequences. The study design included randomly selecting a cohort from those who volunteered to have their own genomes determined. All others (nonvolunteers and those volunteers randomized to not using their own material) would receive an anonymous genome to study. This protocol would allow for a rigorous and meaningful comparison on the effect of a student using their own genome versus an anonymous genome. A number of outcome measures were developed for the students: performance during the new integrated basic science unit (to assess the effect on knowledge), use of Tufts University Sciences Knowledge-based educational material on personalized genetics (to assess the effect of whether using one's own material increases both motivation to learn and interest in the material), and interaction with a standardized patient-actor who would present with his or her genome testing results asking for posttest counseling.

On request, the IRB would informally review research protocols before formal submission to provide guidance regarding potential human subjects' protection and regulatory issues. This informal review was meant to aid investigators in protocol development and anticipate issues that might be raised by the IRB. It was not part of the official review process, and a revised protocol would still need full review. The draft IRB protocol was presented to the Chair of the IRB by S.E. The Chair of the IRB responded with a number of comments, including the following:

1. Given the nature of the proposed protocol, including randomized group assignments, systematic outcome measurements, and the plan to disseminate the findings, the protocol as submitted constituted human subjects research. Given the novel nature of the intervention and potential risks involved, the protocol would not qualify for the exemption from IRB review that is frequently granted for educational research, and the protocol would require IRB review and approval.

2. The proposal did not qualify for a waiver of consent. Informed consent would be required. Both the protocol and the written consent document would need to clearly explain all the potential risks (including a detailed description of the current limitations of the technology) and the purported benefits, such as the opportunity to seek preventive care and lifestyle changes, would have to be explicitly identified as unproven.

3. A clear rationale would have to be stated as to why the investigators thought this program could meaningfully benefit student education and the individual student research subjects. The IRB committee would then weigh these potential benefits against the potential risks.

4. The investigators would need to provide a very detailed plan on how they would maintain privacy as well as monitor and respond to adverse events. In particular, they would need to explicitly address what services would be available for a student who required intervention based on the results of the test, how counseling would be handled (not just for the student but, if necessary, for family members), and from where the resources will come to provide this counseling. 
5. As the study involved a vulnerable population (students) and was being conducted by senior medical school leadership, including deans, appropriate protections against potential coercion would need to be included.

6. The proposal should specify what information would appear in the test results seen by the students. Some thought should be given to limiting the results, especially if this would reduce the "risk" of adverse events.

The results of the IRB discussion suggested that considerably more work would be required to formally submit the protocol. The IRB Chair felt that a revised protocol that adequately addressed these concerns would likely be favorably received by the IRB because the intervention was already readily available to the students outside of the proposed educational research setting.

\section{THE COMPROMISE}

By spring of 2009, several high profile articles had been published describing issues in the early days of personal genomic analyses. ${ }^{3-6}$ The committee decided that it would be wise to begin with anonymous gene profiles rather than the original concept of having students receive their own personal genetic profile. The learning module could then start with the full fall 2009 incoming class and the new curriculum with IRB approval but without the requirement for informed consent. The committee agreed that faculty and students would benefit from the experience and that a significant amount would be learned about both the benefits and limitations of using genetic profiles in the curriculum. One interesting idea that emerged was to use anonymous genomes of individuals known to be at risk for the diseases that are the focus of the first-year curriculum (e.g., cystic fibrosis, human immunodeficiency virus, and diabetes). D.R.W. spoke with 23 andme, who agreed to provide free demonstration accounts for both faculty members and students to access modified anonymous genomes containing disease genotypes.

\section{IMPLEMENTATION}

In late June 2009, the committee held another meeting to discuss integration of the program into the recently revised curriculum. The following actions were agreed on: (1) educational material regarding genomics and personalized genome testing would be added to the curriculum wherever feasible; (2) because the medical genetics course had been moved to the first block of the first year, it provided a logical early venue for the new material; (3) a new lecture would be added to the medical genetics course on the science and technology of genomic testing. This lecture would introduce commercially available personal genome testing, including its limitations regarding analytic and clinical validity; (4) the anonymous genomes provided by 23 andme would be linked to the Tufts University Sciences Knowledge database. A homework assignment would be developed for the students to analyze the results of genome testing on the anonymous patients and to evaluate the clinical validity and utility of the data; (5) the preexisting lecture on ethics would be adapted to include an extensive discussion on the potential benefits and harms associated with personal genome testing as well as the current lack of evidence supporting clinical utility; (6) a protocol would be submitted to the IRB for an educational survey of medical students' attitudes toward personalized genomic testing; and (7) a small group of faculty members would be given the opportunity to undergo personal genomic testing at no charge. They would then discuss their experiences with the committee.

\section{Survey results}

The pregenetics course survey was granted exemption from IRB review and administered in September 2009 to the entire incoming first-year class. The response rate was $97 \%$. The detailed results of the pre- and postcourse surveys will be described in a separate publication. One issue that arose in the course evaluations was that some students were uncomfortable that an individual with industry ties presented material to the class $(23 \%$ of students included an optional comment to this effect). The close association of D.R.W. with Illumina was disclosed before his lecture on genotyping, sequencing, and personalized medicine. In fact, this association was one of the reasons why D.R.W. was invited to give this lecture, as he was uniquely qualified to give students a global perspective on genomic science and technology, as well as an introduction to the techniques, interpretation, benefits, and limitations of commercially available personal genome testing. The students' perception provides an important lesson to institutions contemplating implementing courses and other activities in this area. Conflicts of interest will often be present, and they should not necessarily disqualify someone from participating in the curriculum. However, when such conflicts exist, they must be disclosed to the students. Furthermore, it is important that when students encounter a conflict of interest, a broader discussion should transpire. This discussion took place in the subsequent lecture devoted to ethical issues. In the case in which lecturers with potential conflicts of interest are deliberately included, problems may be averted by specifically explaining the rationale for their inclusion.

\section{Personalized genome testing of faculty members}

Of the seven faculty members who underwent personalized genomic testing by 23 andme, one was diagnosed as being a carrier of mutations in both the cystic fibrosis transmembrane conductance regulator (CFTR) and hereditary hemochromatosis protein precursor (HFE) genes. Another faculty member received results that were consistent with an elevated personal risk of developing glaucoma. The first faculty member will need to have additional testing performed on family members as a result of being diagnosed as a carrier of clinically significant mutations in two different genes. The second faculty member went to an ophthalmologist who specializes in glaucoma to follow-up on the results of testing. That ophthalmologist had no specific knowledge as to how to incorporate the relative risk identified into a care plan but suggested annual screening for glaucoma. This very small sample, using two faculty members who were well aware of the risks and benefits of undergoing testing, illustrates two of the potential dilemmas of providing personalized genetic testing to medical students: (1) the need to involve family members who may not have wanted to be informed that they are at significant risk of having a gene mutation and (2) referral to physicians who lack the education to translate testing results into improved clinical practice.

\section{LESSONS LEARNED}

A report from Stanford University School of Medicine Dean's Newsletter on Genotyping and Education, dated September 28, 2009, (http://deansnewsletter.stanford.edu/archive/ 09_28_09) described a process that was remarkably similar to the one that occurred at Tufts. Given their experiences and our own, we wish to communicate some "lessons learned" and 
recommendations from the process that may be of help to other medical schools considering the implementation of personalized genetics into the curriculum.

1. Before offering students the opportunity to have personalized genetic testing, the issue should be considered by a high-level faculty committee with broad multidisciplinary representation.

2. The IRB should be engaged as early as possible in the process regarding issues related to offering personalized genomic testing to students in an educational research setting.

\section{RECOMMENDATIONS}

1. If an institution is going to offer testing to trainees, a plan should be made regarding both protection of privacy and follow-up of abnormal tests. Students should be told in advance of testing where to go for counseling regarding abnormal results and who will pay for such counseling.

2. Curriculum committees should explore ways of enriching educational content in the curriculum with genetics, genomics, genome-wide association studies, and sequencing using anonymous or publicly accessible genomes. Discussion of the benefits, limitations, and potential harms of such testing should be an integral part of the educational process.

3. When deliberately including lecturers with potential conflicts of interest, consideration should be given to includ- ing an explanation of the decision to include the specific lecturer despite the potential conflict.

Medical school education regarding genomic analysis and personalized medicine is a necessity, both to be able to translate the advances made by advances in genetic analysis and knowledge into improvements in human health and to begin to think of diseases as disruptions in specific pathways. Our experiences illustrate that adding this material to a medical school curriculum is a complex process that deserves careful thought and broad discussion within the academic community.

\section{ACKNOWLEDGMENTS}

David R. Walt is a co-founder of Illumina. He holds equity in Illumina. Diana W. Bianchi chairs the Clinical Advisory Board of Artemis Health, Inc. She holds stock options and receives honoraria as a consultant for Artemis Health, Inc.

\section{REFERENCES}

1. Salari K. The dawning era of personalized medicine exposes a gap in medical education. PLoS Med 2009;6:e1000138.

2. Guttmacher AE, Porteous ME, McInerney JD. Educating health-care professionals about genetics and genomics. Nat Rev Genet 2007;8:151-157.

3. Burke W, Psaty BM. Personalized medicine in the era of genomics. JAMA 2007;298:1682-1684

4. Janssens AC, van Duijn CM. Genome-based prediction of common diseases: advances and prospects. Hum Mol Genet 2008;17:R166-R173.

5. Janssens AC, Gwinn M, Bradley LA, Oostra BA, van Duijn CM, Khoury MJ. A critical appraisal of the scientific basis of commercial genomic profiles used to assess health risks and personalize health interventions. Am J Hum Genet 2008;82:593-599.

6. Fox JL. What price personal genome exploration? Nat Biotechnol 2008;26: $1105-1108$ 